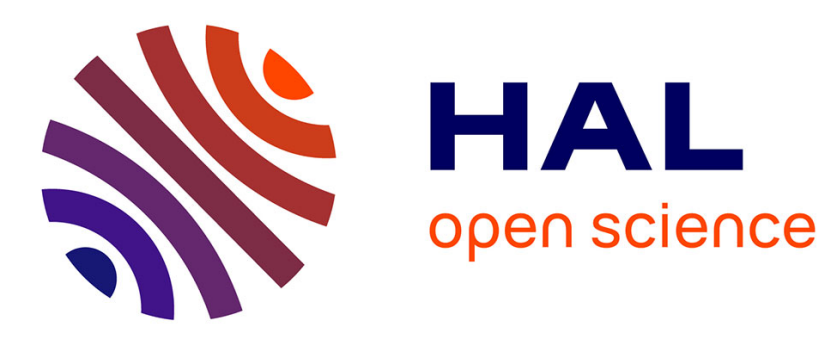

\title{
Adaptive Constructive Interval Disjunction
}

Bertrand Neveu, Gilles Trombettoni

\section{To cite this version:}

Bertrand Neveu, Gilles Trombettoni. Adaptive Constructive Interval Disjunction. ICTAI: International Conference on Tools with Artificial Intelligence, Nov 2013, Washington, DC, United States. pp.900-906, 10.1109/ICTAI.2013.138 . hal-00936654

\section{HAL Id: hal-00936654 \\ https://hal-enpc.archives-ouvertes.fr/hal-00936654}

Submitted on 27 Jan 2014

HAL is a multi-disciplinary open access archive for the deposit and dissemination of scientific research documents, whether they are published or not. The documents may come from teaching and research institutions in France or abroad, or from public or private research centers.
L'archive ouverte pluridisciplinaire HAL, est destinée au dépôt et à la diffusion de documents scientifiques de niveau recherche, publiés ou non, émanant des établissements d'enseignement et de recherche français ou étrangers, des laboratoires publics ou privés. 


\section{Adaptive Constructive Interval Disjunction}

\author{
Bertrand Neveu \\ LIGM \\ Université Paris Est \\ Marne-la-Vallée, France \\ Email: Bertrand.Neveu@enpc.fr
}

\author{
Gilles Trombettoni \\ LIRMM \\ Université Montpellier II \\ Montpellier, France \\ Email: Gilles.Trombettoni@lirmm.fr
}

\begin{abstract}
An operator called $C I D$ and an efficient variant $3 B C I D$ were proposed in 2007. For numerical CSPs handled by interval methods, these operators compute a partial consistency equivalent to Partition-1-AC for discrete CSPs. The two main parameters of $C I D$ are the number of times the main $C I D$ procedure is called and the maximum number of sub-intervals treated by the procedure. The 3BCID operator is state-of-theart in numerical CSP solving, but not in constrained global optimization.

This paper proposes an adaptive variant of $3 B C I D$. The number of variables handled is auto-adapted during the search, the other parameters are fixed and robust to modifications. On a representative sample of instances, $A C I D$ appears to be the best approach in solving and optimization, and has been added to the default strategies of the Ibex interval solver.
\end{abstract}

\section{Constructive interval Disjunction (CID)}

A filtering/contracting operator for numerical CSPs called Constructive Interval Disjunction (in short CID) has been proposed in [13]. Applied first to continuous constraint satisfaction problems handled by interval methods, it has been more recently applied to constrained global optimization problems. This algorithm is state-of-the-art in constraint satisfaction, but is generally dominated by constraint propagation algorithms like $\mathrm{HC} 4$ in optimization. The main practical contribution is that an adaptive version of CID becomes efficient for both real-valued satisfaction and optimization problems, while needing no additional parameter value from the user.

\section{A. Shaving}

The shaving principle is used to compute the Singleton Arc Consistency (SAC) of finite domain CSPs [7] and the 3B-consistency of numerical CSPs [9]. It is also at the core of the SATZ algorithm [11] used to prove the satisfiability of Boolean formula. Shaving works as follows. A value is temporarily assigned to a variable (the other values are temporarily discarded) and a partial consistency is computed on the remaining subproblem. If an inconsistency is obtained then the value can be safely removed from the domain of the variable. Otherwise, the value is kept in the domain.

Contrarily to arc consistency, this consistency is not incremental [7]. Indeed, the work of the underlying refutation procedure on the whole subproblem is the reason why a single value can be removed. Thus, obtaining the singleton arc consistency on finite-domain CSPs requires an expensive fixed-point algorithm where all the variables must be handled again every time a single value is removed [7]. The remark still holds for the improved version SAC-Opt [5]. A similar idea can be followed on numerical CSPs (NCSPs).

\section{B. Numerical CSP}

An NCSP is defined by a tuple $P=(X,[X], C)$, where $X$ denotes a $n$-set of numerical, real-valued variables ranging in a domain $[X]$. We denote by $\left[x_{i}\right]=\left[x_{i}, \overline{x_{i}}\right]$ the interval/domain of variable $x_{i} \in X$, where $x_{i}, \overline{\overline{x_{i}}}$ are floating-point numbers (allowing interval algorithms to be implemented on computers). A solution of $P$ is an $n$-vector in $[X]$ satisfying all the constraints in $C$. The constraints defined in an NCSP are numerical. They are equations and inequalities using mathematical operators like $+, \cdot, /$, exp, $\log$, sin.

A Cartesian product of intervals like the domain $[X]=$ $\left[x_{1}\right] \times \ldots \times\left[x_{n}\right]$ is called a (parallel-to-axes) box. $w\left(x_{i}\right)$ denotes the width $\overline{x_{i}}-x_{i}$ of an interval $\left[x_{i}\right]$. The width of a box is given by the width $\overline{x_{m}}-x_{m}$ of its largest dimension $x_{m}$. The union of several boxes is generally not a box, and a Hull operator has been defined instead to define the smallest box enclosing all of them.

NCSPs are generally solved by a Branch \& Contract interval strategy:

- Branch: a variable $x_{i}$ is chosen and its interval $\left[x_{i}\right]$ is split into two sub-intervals, thus making the whole process combinatorial.

- Contract: a filtering process allows contracting the intervals (i.e., improving interval bounds) without loss of solutions.

The process starts with the initial domain $[X]$ and stops when the leaves/boxes of the search tree reach a width inferior to a precision given as input. These leaves yield an approximation of all the solutions of the NCSP.

Several contraction algorithms have been proposed. Let us mention the constraint propagation algorithm called $\mathrm{HC} 4$ [3], [10], an efficient implementation of 2B [9], that can enforce the optimal local consistency (called hull-consistency) only if strong hypotheses are met (in particular, each variable 
must occur at most once in a same constraint). The $2 \mathrm{~B}-$ Revise procedure works with all the projection functions of a given constraint. Informally, a projection function isolates a given variable occurrence within the constraint. For instance, consider the constraint $x+y=z . x ; x \leftarrow z . x-y$ is a projection function (among others) that aims at reducing the domain of variable $x$. Evaluating the projection function with interval arithmetics on the domain $[x] \times[y] \times[z]$ (i.e., replacing the variable occurrences of the projection function by their domains and using the interval counterpart of the involved mathematical operators) provides an interval that is intersected with $[x]$. Hence a potential domain reduction. A constraint propagation loop close to that of AC3 is used to propagate reductions obtained for a given variable domain to the other constraints in the system.

\section{3 B algorithm}

Stronger interval partial consistencies have also been proposed. 3B-consistency [9] is a theoretical partial consistency similar to SAC for CSP although limited to the bounds of the domains. Consider the $2 n$ subproblems of the studied NCSP where each interval $\left[x_{i}\right](i \in\{1 . . n\})$ is reduced to its lower bound $x_{i}$ (resp. upper bound $\overline{x_{i}}$ ). 3B-consistency is enforced iff each of these $2 n$ subproblems is hull-consistent.

In practice, the $3 \mathrm{~B}(w)$ algorithm splits the intervals in several sub-intervals, also called slices, of width $w$, which gives the accuracy: the $3 \mathrm{~B}(\mathrm{w})$-consistency is enforced iff the slices at the bounds of the handled box cannot be eliminated by $\mathrm{HC} 4$. Let us denote $\operatorname{var} 3 \mathrm{~B}$ the procedure of the $3 \mathrm{~B}$ algorithm that shaves one variable interval $\left[x_{i}\right]$ and $s_{3 b}$ its parameter, a positive integer specifying a number of subintervals: $w=w\left(x_{i}\right) / s_{3 b}$ is the width of a sub-interval.

\section{D. $C I D$}

Constructive Interval Disjunction (CID) is a partial consistency stronger than 3B-consistency [13]. CID-consistency is similar to Partition-1-AC (P-1-AC) in finite domain CSPs [4]. P-1-AC is strictly stronger than SAC [4].

The main procedure varCID handles a single variable $x_{i}$. The main parameters of varCID are $x_{i}$, a number $s_{c i d}$ of sub-intervals (accuracy) and a contraction algorithm ctc like HC4. $\left[x_{i}\right]$ is split into $s_{c i d}$ slices of equal width, each corresponding subproblem is contracted by the contractor ctc and the hull of the different contracted subproblems is finally returned, as shown in Algorithm 1.

Intuitively, CID generalizes $3 \mathrm{~B}$ because a sub-box that is eliminated by $\operatorname{var} 3 \mathrm{~B}$ can also be discarded by varCID. In addition, contrary to $\operatorname{var} 3 \mathrm{~B}$, varCID can also contract $[X]$ along several dimensions.

Note that in the actual implementation the for loop can be interrupted earlier, when $[X]^{\prime}$ becomes equal to the initial box $[X]$ in all the dimensions except $x_{i}$.

var3BCID is a hybrid and operational variant of varCID.

$$
\begin{aligned}
& \text { Procedure } \operatorname{VarCID}\left(x_{i}, s_{c i d},(X, C \text {, in-out }[X]), \text { ctc }\right) \\
& \qquad \begin{array}{l}
{[X]^{\prime} \leftarrow \text { empty box }} \\
\text { for } j \leftarrow 1 \text { to } s_{c i d} \text { do } \\
\quad{ }^{*} \text { The } j^{t h} \text { sub-box of }[X] \text { on } x_{i} \text { is handled: */ } \\
\text { sliceBox } \leftarrow \text { SubBox }\left(j, x_{i},[X]\right) \\
/ * \text { Enforce a partial consistency on the sub-box: */ } \\
\text { sliceBox' } \leftarrow \text { ctc }(X, C, \text { sliceBox }) \\
/ * \text { 'Union" with previous sub-boxes: */ } \\
{[X]^{\prime} \leftarrow \operatorname{Hul}\left([X]^{\prime}, \text { sliceBox' }\right)} \\
{[X] \leftarrow[X]^{\prime}}
\end{array}
\end{aligned}
$$

Algorithm 1: The main VarCID procedure of the CID operator shaving a given variable $x_{i}$.

1) Like $\operatorname{var} 3 \mathrm{~B}$, it first tries to eliminate sub-intervals at the bounds of $\left[x_{i}\right]$ of width $w=w\left(x_{i}\right) / s_{3 b}$ each. We store the left box $\left[X_{l}\right]$ and the right box $\left[X_{r}\right]$ that are not excluded by the contractor ctc (if any).

2) Second, the remaining box $[X]^{\prime}$ is handled by varCID that splits $[X]^{\prime}$ into $s_{\text {cid }}$ sub-boxes. The subboxes are contracted by $c t c$ and hulled, giving $\left[X_{c i d}\right]$.

3 ) Finally, we return the hull of $\left[X_{l}\right],\left[X_{r}\right]$ and $\left[X_{c i d}\right]$.

The var3BCID process is illustrated in Figure 1.

3B:

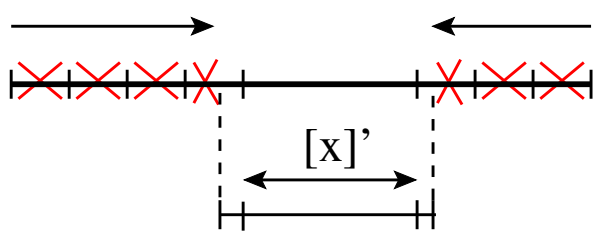

CID:

Figure 1. Task of the var3BCID procedure. The parameter $s_{3 b}$ is set to 10 and $s_{\text {cid }}$ is set to 1 .

var3BCID comes from the wish of managing different widths (accuracies) for $s_{3 b}$ and $s_{c i d}$. Indeed, the best choice for $s_{3 b}$ generally belongs to $\{5 . .20\}$ while $s_{c i d}$ should always be set to 1 or 2 (implying a final hull of 3 or 4 sub-boxes). The reason is that the actual time cost of the shaving part is smaller than the one of the constructive domain disjunction. Indeed, if no sub-interval is discarded by var3B, only two calls to $c t c$ are performed, one for each bound of the handled interval; if varCID is applied, the subcontractor is often called $s_{\text {cid }}$ times.

The procedure var3BCID has been deeply studied and experimented in the past. The number and the order in which calls to var 3BCID are achieved is a harder question studied in this paper.

\section{AdAPTIVE CID: LEARNING THE NUMBER OF HANDLED VARIABLES}

Like for $S A C$ or $3 B$, a quasi fixed-point in terms of contraction can be reached by $3 B C I D$ (or CID) by calling var3BCID inside two nested loops. An inner loop calls var3BCID on each variable $x_{i}$. An outer loop calls the 
inner loop until no interval is contracted more than a predefined (width) precision (thus reaching a quasi-fixed point). Let us call $3 B C I D-f p$ (fixed-point) this historical version.

Two reasons led us to radically change this policy. First, as said above, var3BCID can contract the handled box in several dimensions. One significant advantage is that the fixed-point in terms of contraction can thus be reached in a small number of calls to var3BCID. On most of the instances in satisfaction or optimization, it appears that a quasi fixed-point is reached in less than $n$ calls. In this case, 3BCID is clearly too expensive. Second, the varCID principle is close to a branching point in a search tree. The difference is that a hull is achieved at the end of the sub-box contractions. Therefore an idea is to use a standard branching heuristic to select the next variable to be "varcided". We will write in the remaining part of the paper that a variable is varcided when the procedure var3BCID is called on that variable to contract the current box.

To sum up, the idea for rendering $3 B C I D$ even more efficient in practice is to replace the two nested loops by a single loop calling numVarCID times var3BCID and to use an efficient variant of the Smear function branching heuristic for selecting the variables to be varcided (called SmearSumRel in [12]). Informally, the Smear function favors variables having a large domain and a high impact on the constraints - measuring interval partial derivatives.

A first idea is to fix numVarCID to the number $n$ of variables. We call $3 B C I D-n$ this version. This gives good results in satisfaction but is dominated by pure constraint propagation in optimization. As said above, it is too time costly when the right numVarCID is smaller than $n$ (which is often the case in optimization), but can also have a very bad impact on performance if a bigger effort brought a significantly greater filtering.

The goal of Adaptive CID (ACID) is precisely to compute dynamically during search the value of the numVarCID parameter. Several auto-adaptation policies have been tested and we report three interesting versions. All the policies measure the decrease in search space size after each call to var3BCID. They measure a contraction ratio of a box $[X]^{b}$ over another box $[X]^{a}$ as an average relative gain in all the dimensions:

$$
\text { gainRatio }\left([X]^{b},[X]^{a}\right)=\frac{1}{n} \sum_{i=1}^{n}\left(1-\frac{w\left(x_{i}^{b}\right)}{w\left(x_{i}^{a}\right)}\right)
$$

\section{A. ACID0: auto-adapting numVarCID during search}

The first version ACIDO adapts the number of shaved variables dynamically at each node of the search tree. First, the variables are sorted by their impact, computed by the same formula as the SmearSumRel function (used for branching). Variables are then varcided until the cumulative contraction ratio during the last $n v$ calls to var3BCID becomes less than ctratio. This algorithm has thus 2 parameters $n v$ and ctratio, and it was difficult to tune them. We experimentally found that ctratio could be fixed to 0.001 and $n v$ should depend on the number of variables $n$ of the problem. Setting $n v$ to 1 is often a bad choice, and fixing it with the formula $n v=\max \left(3, \frac{n}{4}\right)$ experimentally gave the best results. The experimental results are not bad but this policy prevents numVarCID from reaching 0 , i.e. from calling only constraint propagation. This is a significant drawback when a simple constraint propagation is the most efficient approach.

\section{B. ACID1: interleaving learning and exploitation phases}

A more sophisticated approach avoids this drawback. ACID 1 interleaves learning and exploitation phases for autoadapting the numVarCID value. Depending on the node number, the algorithm is in a learning or in an exploitation phase.

The behavior of ACID1, shown in Algorithm 2, is the following:

- The variables are first sorted according to their impact measurement (using the SmearSumRel heuristic).

- During a learning phase (during learnLength nodes), we then analyze how the contraction ratio evolves from a var3BCID call to the next one, and store the number $k v a r C I D$ of varcided variables necessary to obtain most of the possible filtering.

More precisely, 2.numVarCID variables are varcided at each node (with a minimum value equal to 2 , in case num $\operatorname{Var} C I D=0$ ). In the first learning phase, we handle $n$ variables. At the current node, the lastSignificantGain function returns the number $k v a r C I D$ of varcided variables giving the last significant improvement. After the $k_{\text {varCID }}{ }^{\text {th }}$ call to var3BCID, the gain in current box size from a var3BCID call to the next one, computed by the gainRatio formula, never exceeded a small given ratio, called ctratio. This analysis starts from the last varcided variable. (For the readibility of the pseudocode, we omit the parameters of the var3BCID procedure, i.e. $s_{3 b}, s_{c i d}$, the constraints $C$ and the contractor ctc.)

- During the exploitation phase following the previous learning phase, the average of the different kvarCID values (obtained in the nodes of the learning phase) provides the new value of numVarCID. This value will be used by 3 BCID during the exploitation phase. Compared to the previous value (previous call to an exploitation phase), note that this new value can at most double, but can also drastically decrease.

Every cycleLength nodes in the search tree, both phases are called again.

Numerous variants of this schema were tested. In particular, it is counterproductive to learn numVarCID only once 


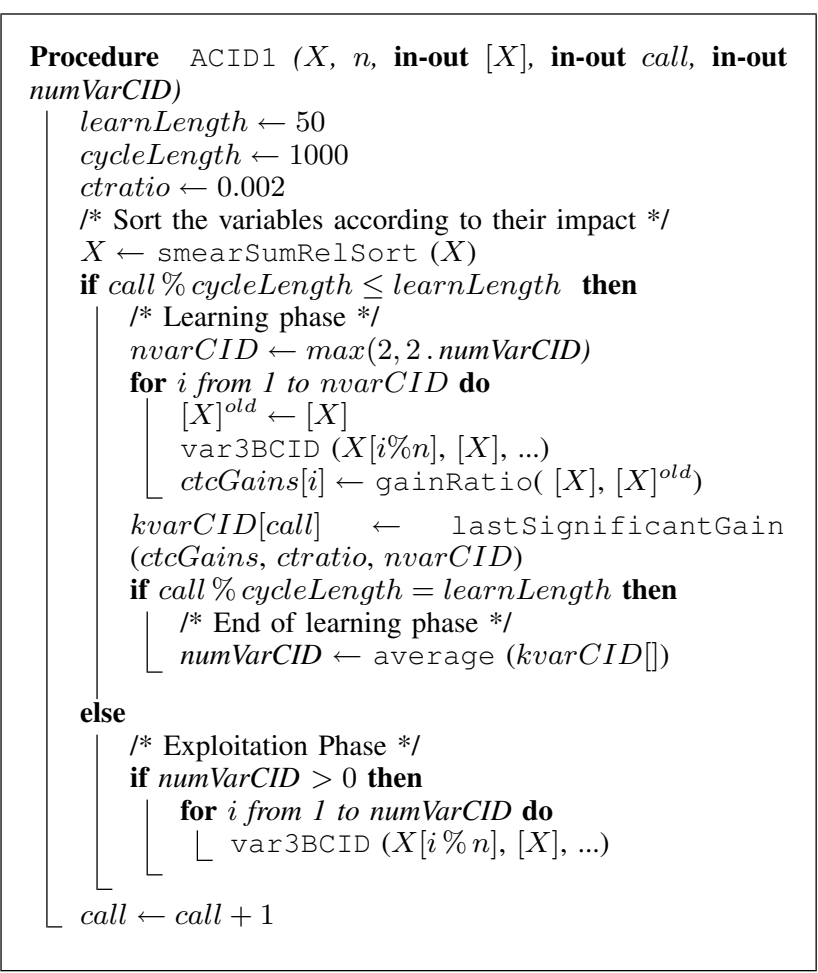

Algorithm 2: Algorithm ACID1

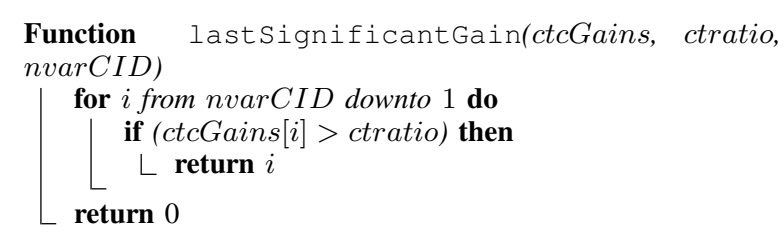

or, on the contrary, to memorize the computations from a learning phase to another one.

We fixed experimentally the 3 parameters of the ACID 1 procedure learnLength, cycleLength and ctratio, respectively to 50, 1000 and 0.002 . ACID 1 becomes then a parameter free procedure. With these parameter values, the overhead of the learning phases (where we double the previous numVarCID value) remains small.

\section{ACID2: taking into account the level in the search tree}

A criticism against ACID1 is that we average kvarCID values obtained at different levels of the search tree. This drawback is partially corrected by the successive learning phases of ACID1, where each learning phase corresponds to a part of the search tree.

In order to go further in that direction, we designed a refinement of ACID1 for which each learning phase tunes at most 10 different values depending on the width of the studied box. A value corresponds to one order of magnitude in the box width. For example, we store a numVarCID value for the boxes with a width comprised between 1 and 0.1 , another one for the boxes with a width comprised between 0.1 and 0.01 , etc. However, this approach, called ACID2, gave in general results similar to those of ACID1 and appeared to be less robust. Indeed, only a few nodes sometimes fall at certain width levels, which renders the statistics not significant.

\section{EXPERIMENTS}

All the algorithms were implemented in the $\mathrm{C}++$ interval library Ibex (Interval Based EXplorer) [6]. All the experiments were run on the same computer (Intel X8 $63 \mathrm{GHz}$ ). We tested the algorithms on square NCSP solving and constrained global optimization. NCSP solving consists in finding all the solutions of a square system of $n$ nonlinear equations with $n$ real-values variables with bounded domains. Global optimization consists in finding the global minimum of a function over $n$ variables subject to constraints (equations and inequalities), the objective function and/or the constraints being non-convex.

\section{A. Experiments in constraint satisfaction}

We selected from the COPRIN benchmark ${ }^{1}$ all the systems that were solved by one of the tested algorithms in a time comprised between $2 \mathrm{~s}$ and $3600 \mathrm{~s}$. The timeout was fixed to $10,000 \mathrm{~s}$. The required precision on the solution is $10^{-8}$. Some of these problems are scalable. In this case, we selected the problem with the greatest number of variables that was solved by one of the algorithms in less than one hour.

We compared our ACID method and its variants with the well known filtering techniques: a simple constraint propagation HC4, 3BCID-n (see Section II) and 3BCID-fp (fixed-point) in which a new iteration on all the variables is run when a variable domain width is reduced by more than $1 \%$. At each node of the search tree, we used the following sequence of contractors : $\mathrm{HC} 4$, shaving, Interval-Newt on [8], and X-Newt on [2]. shaving denotes a variant of $A C I D, 3 B C I D-n, 3 B C I D-f p$ or nothing when only $\mathrm{HC} 4$ is tested.

For each problem, we used the best bisection heuristics available (among two variants of the Smear function [12]). The main parameter ctratio of ACID1 and ACID2, measuring a stagnation in the filtering while variables are varcided, was fixed to 0.002 . The var3BCID parameters $s_{3 b}$ and $s_{\text {cid }}$ were fixed to the default settings, respectively 10 and 1, proposed in [13]. Experiments on the selected instances confirm that these settings are relevant and robust to variations. In particular, setting $s_{3 b}$ to 10 gives results better than with smaller values $\left(s_{3 b}=5\right)$ and with greater values. (For 21 over the 26 instances, $s_{3 b}=20$ gives worse results.) As

\footnotetext{
${ }^{1}$ www-sop.inria.fr/coprin/logiciels/ALIAS/Benches/benches.html
} 
Table I

CONTINUOUS CSP SOLVING: ACID1 RESULTS. FOR EACH PROBLEM, WE PRESENT ITS NUMBER OF VARIABLES AND THE RESULTS OBTAINED BY ACID 1: THE CPU TIME, THE NUMBER OF BRANCHING NODES IN THE SEARCH TREE, THE AVERAGE NUMBER OF VARCIDED VARIABLES (TUNED BY ACID1 DYNAMICALLY). WE ALSO REPORT THE BEST AND THE WORST METHODS AMONG ACID1, HC 4, 3BCID-fP, AND 3BCID-n, THE CPU TIME RATIO OF ACID1 OVER THE BEST METHOD AND OVER THE WORST METHOD.

\begin{tabular}{|c|c|c|c|c|c|c|c|c|}
\hline & \#var & ACID1 & ACID1 & ACID 1 & best & worst & Time ratio & Time ratio \\
\hline & & time & \#nodes & \#varcids & & & ACID 1/best & ACID1/worst \\
\hline Bellido & 9 & 3.45 & 518 & 5 & ACID 1 & $\mathrm{HC} 4$ & 1 & 0.89 \\
\hline Brown-7 & 7 & 396 & 540730 & 4.5 & ACID 1 & $\mathrm{HC} 4$ & 1 & 0.82 \\
\hline Brent-10 & 10 & 17.6 & 3104 & 9 & ACID1 & $\mathrm{HC} 4$ & 1 & 0.14 \\
\hline Butcher8a & 8 & 981 & 204632 & 9 & $3 B C I D-n$ & $\mathrm{HC} 4$ & 1.03 & 0.49 \\
\hline Butcher8b & 8 & 388 & 93600 & 10.8 & ACID1 & $\mathrm{HC} 4$ & 1 & 0.31 \\
\hline Design & 9 & 29.2 & 5330 & 11 & $3 B C I D-n$ & $\mathrm{HC} 4$ & 1.07 & 0.37 \\
\hline Dietmaier & 12 & 926 & 82364 & 26.3 & ACID 1 & $\mathrm{HC} 4$ & 1 & 0.19 \\
\hline Directkin & 11 & 32.7 & 2322 & 7 & ACID 1 & $3 B C I D-f p$ & 1 & 0.84 \\
\hline Disc.integralf2-16 & 32 & 592 & 58464 & 0.4 & $\mathrm{HC} 4$ & $3 B C I D-f p$ & 1.02 & 0.52 \\
\hline Eco-12 & 11 & 3156 & 297116 & 12 & ACID 1 & $\mathrm{HC} 4$ & 1 & 0.32 \\
\hline Fredtest & 6 & 25.2 & 11480 & 0.8 & $\mathrm{HC} 4$ & $3 B C I D-f p$ & 1.04 & 0.91 \\
\hline Fourbar & 4 & 437 & 183848 & 0.1 & ACID 1 & $3 B C I D-n$ & 1 & 0.85 \\
\hline Geneig & 6 & 178 & 83958 & 2.9 & $\mathrm{HC} 4$ & $3 B C I D-f p$ & 1.02 & 0.82 \\
\hline Hayes & 7 & 3.96 & 1532 & 7.5 & $3 B C I D-n$ & $\mathrm{HC} 4$ & 1.14 & 0.77 \\
\hline I5 & 10 & 15.9 & 3168 & 11.5 & ACID 1 & $\mathrm{HC} 4$ & 1 & 0.13 \\
\hline Katsura-25 & 26 & 691 & 5396 & 10.4 & ACID 1 & $3 B C I D-f p$ & 1 & 0.67 \\
\hline Pramanik & 3 & 23.1 & 23696 & 0.2 & ACID 1 & $\mathrm{HC} 4$ & 1 & 0.69 \\
\hline Reactors-42 & 42 & 1285 & 23966 & 134 & $3 B C I D-f p$ & $\mathrm{HC} 4$ & 1.07 & 0.13 \\
\hline Reactors $2-30$ & 30 & 1220 & 38136 & 90 & $3 \mathrm{BCID}-\mathrm{n}$ & $\mathrm{HC} 4$ & 1.14 & 0.12 \\
\hline Synthesis & 33 & 356 & 7256 & 53.8 & 3BCID-fp & $\mathrm{HC} 4$ & 1.15 & 0.25 \\
\hline Trigexp2-23 & 23 & 2530 & 227136 & 39.4 & $3 B C I D-f p$ & $\mathrm{HC} 4$ & 1.26 & 0.25 \\
\hline Trigo1-18 & 18 & 2625 & 37756 & 6.1 & ACID 1 & $3 B C I D-f p$ & 1 & 0.8 \\
\hline Trigolsp-35 & 36 & 2657 & 70524 & 2.4 & ACID 1 & $3 B C I D-f p$ & 1 & 0.41 \\
\hline Virasoro & 8 & 1592 & 266394 & 0.6 & $3 B C I D-n$ & $3 B C I D-f p$ & 1.08 & 0.28 \\
\hline Yamamura1-16 & 16 & 2008 & 68284 & 0.4 & $3 B C I D-n$ & $\mathrm{HC} 4$ & 1.02 & 0.86 \\
\hline Yamamura1sp-500 & 501 & 1401 & 146 & 144 & ACID1 & $\mathrm{HC} 4$ & 1 & 0.14 \\
\hline
\end{tabular}

shown in Table I, ACID1 appears to be often the best one, or close to the best one. In only 4 problems on 26 , it was more than $10 \%$ slower than the best. The number of varcided variables was tuned close to 0 in the problems where $\mathrm{HC} 4$ was sufficient, and more than the number of variables in the problems where 3BCID-fp appeared to be the best method.

In the left part of Table II, we summarize the results obtained by the three variants of ACID and their competitors. It appears that only ACID 1 could solve the 26 problems in 1 hour, while HC 4 could solve only 21 problems in 10,000s. The gains in cpu time obtained by ACID1 w.r.t. competitors are sometimes significant (see the line max gain), while its losses remain weak. ACIDO with its two parameters was more difficult to tune, and it was not interesting to run the more complex algorithm ACID2. ACID1 obtains better gains w.r.t $3 B C I D-n$ in total time than on average because the best gains were obtained on difficult instances with more variables. In the right part of the table, we report the solving time ratios obtained when $\mathrm{X}$-Newt on is removed $(\neg \mathrm{XN})$ from the contractor sequence (4 problems could not be solved in 10,000s). The only ACID variant studied was ACID1. ACID1 and 3BCID-n obtain globally similar results, better than $3 B C I D-f p$, but with a greater dispersion (i.e., standard deviation) than with $\mathrm{X}$-Newton since the shaving takes a more important part in the contraction.

\section{B. Experiments in constrained global optimization}

We selected in the series 1 of the Coconut constrained global optimization benchmark ${ }^{2}$ all the 40 instances that ACID or a competitor could solve in a CPU time comprised between $2 \mathrm{~s}$ and $3600 \mathrm{~s}$.

The time out was fixed to 3600s. We used the IbexOpt strategy of Ibex that performs a Best First Branch \& Bound. The experimental protocol is the same as the NCSP experimental protocol, except that we do not use Interval-Newton that is only implemented for square systems.

For each instance, we use the best bisection heuristics (the same for all methods) among largestFirst, roundRobin and variants of the Smear function. The precision required on the objective is $10^{-8}$. Each equation is relaxed by two inequalities with a precision $10^{-8}$.

Table III reports the same columns as Table I, plus a column indicating the number of constraints of the instance. For the constraint programming part of IbexOpt, HC 4 is state of the art and 3BCID is rarely needed in optimization. ${ }^{3}$

\footnotetext{
${ }^{2}$ www.mat.univie.ac.at/ $\sim$ neum/glopt/coconut/Benchmark/Benchmark. html

${ }^{3}$ In fact, the more recent Mohc constraint propagation algorithm [1] is better than HC4. Mohc is not yet reimplemented in Ibex 2.0. However, 3BCID (MohC) shows roughly the same gains w.r.t. Mohc than 3BCID (HC4) does w.r.t. HC 4...
} 
Table II

NCSP: SOLVING TIME GAIN RATIOS. WE REPORT THE NUMBER OF PROBLEMS SOLVED BEFORE 3600 S AND BEFORE 10,000 S, AND DIFFERENT STATISTICS ON THE CPU TIME GAIN RATIO OF ACID1 OVER EACH COMPETITOR $C_{i}$ (ONE PER COLUMN): THE AVERAGE, MAXIMUM, MINIMUM AND STANDARD DEVIATION VALUES OF THIS RATIO $\frac{\text { acid1 time }}{C_{i} \text { time }}$

\begin{tabular}{|c|c|c|c|c|c|c|c|c|c|}
\hline & ACID1 & $\mathrm{HC} 4$ & 3BCID-fp & $3 B C I D-n$ & ACID 0 & ACID2 & ACID1 & $3 B C I D-f p$ & 3BCID-n \\
\hline & & & & & & & $\neg \mathrm{XN}$ & $\neg \mathrm{XN}$ & $\neg \mathrm{XN}$ \\
\hline \#solved instances $<3600$ & 26 & 20 & 23 & 24 & 25 & 24 & 20 & 16 & 20 \\
\hline \#solved instances $<10000$ & 26 & 21 & 26 & 26 & 26 & 26 & 22 & 21 & 22 \\
\hline Average gain & 1 & 0.7 & 0.83 & 0.92 & 0.96 & 0.91 & 1 & 0.78 & 1.02 \\
\hline Maximum gain & 1 & 0.13 & 0.26 & 0.58 & 0.45 & 0.48 & 1 & 0.18 & 0.38 \\
\hline Maximum loss & 1 & 1.04 & 1.26 & 1.14 & 1.23 & 1.05 & 1 & 2.00 & 1.78 \\
\hline Standard deviation gain & 0 & 0.32 & 0.23 & 0.15 & 0.15 & 0.19 & 0 & 0.34 & 0.28 \\
\hline Total time & 23594 & $>72192$ & 37494 & 27996 & 26380 & 30428 & 29075 & 50181 & 31273 \\
\hline Total gain & 1 & & 0.63 & 0.84 & 0.89 & 0.78 & 1 & 0.58 & 0.93 \\
\hline
\end{tabular}

Therefore, we report in the penultimate column a comparison between ACID1 and HC4. The number of varcided variables was indeed tuned by ACID1 to a value comprised between 0 and the number of variables. Again, we can see that ACID1 is robust and is the best, or at most $10 \%$ worse than the best, for 34 among 40 instances. Table IV shows that we obtained an average gain of $10 \%$ over HC4. It is significant because the CP contraction is only a part of the IbexOpt algorithm [12] (linear relaxation and the search of feasible points are other important parts, not studied in this paper and set to their default algorithms in IbexOpt). ACID 0 shaves a minimum of 3 variables, which is often too much. ACID2 obtains results slightly worse than ACID1, rendering this refinement not promising in practice.

\section{CONCLUSION}

We have presented in this paper an adaptive version of the 3BCID contraction operator used by interval methods and close to partition-1-AC. The best variant of this Adaptive CID operator (ACID1 in the paper) interleaves learning phases and exploitation phases to auto-adapt the number of variables handled. These variables are selected by an efficient branching heuristic and all the other parameters are fixed and robust to modifications.

Overall, ACID 1 adds no parameter to the solving or optimization strategies. It offers the best results on average and is the best or close to the best on every tested instance, even in presence of the best Ibex devices (Interval-Newton, $X$-Newton). Therefore ACID1 has been added to the Ibex default solving and optimization strategies.

\section{REFERENCES}

[1] I. Araya, G. Trombettoni, and B. Neveu. Exploiting Monotonicity in Interval Constraint Propagation. In Proc. AAAI, pages $9-14,2010$.

[2] I. Araya, G. Trombettoni, and B. Neveu. A Contractor Based on Convex Interval Taylor. In Proc. CPAIOR, volume 7298 of $L N C S$, pages 1-16. Springer, 2012.
[3] F. Benhamou, F. Goualard, L. Granvilliers, and J.-F. Puget. Revising Hull and Box Consistency. In Proc. ICLP, volume 5649 of $L N C S$, pages 230-244. Springer, 1999.

[4] H. Bennaceur and M.-S. Affane. Partition-k-AC: An Efficient Filtering Technique Combining Domain Partition and Arc Consistency. In Proc. CP, volume 2239 of LNCS, pages 560564. Springer, 2001.

[5] C. Bessiere and R. Debruyne. Optimal and Suboptimal Singleton Arc Consistency Algorithms. In Proc. IJCAI, pages 54-59, 2005.

[6] G. Chabert and L. Jaulin. Contractor Programming. Artificial Intelligence, 173:1079-1100, 2009.

[7] R. Debruyne and C. Bessiere. Some Practicable Filtering Techniques for the Constraint Satisfaction Problem. In Proc. IJCAI, pages 412-417, 1997.

[8] E. Hansen. Global Optimization using Interval Analysis. Marcel Dekker inc., 1992.

[9] O. Lhomme. Consistency Techniques for Numeric CSPs. In Proc. IJCAI, pages 232-238, 1993.

[10] F. Messine. Méthodes d'optimisation globale basées sur l'analyse d'intervalle pour la résolution des problèmes avec contraintes. PhD thesis, LIMA-IRIT-ENSEEIHT-INPT, Toulouse, 1997.

[11] C. Min Li and Anbulagan. Heuristics Based on Unit Propagation for Satisfiability Problems. In Proc. IJCAI, pages 366-371, 1997.

[12] G. Trombettoni, I. Araya, B. Neveu, and G. Chabert. Inner Regions and Interval Linearizations for Global Optimization. In Proc. AAAI, pages 99-104, 2011.

[13] G. Trombettoni and G. Chabert. Constructive Interval Disjunction. In Proc. CP, volume 4741 of $L N C S$, pages 635-650. Springer, 2007. 
Table III

OPTIMIZATION PROBLEMS: ACID1 RESULTS

\begin{tabular}{|c|c|c|c|c|c|c|c|c|c|c|}
\hline & \#var & $\#$ ctr & ACID1 & ACID1 & ACID1 & best & worst & Time ratio & Time ratio & Time ratio \\
\hline & & & time & \#nodes & \#varcids & & & ACID1/best & $\mathrm{ACID} 1 / \mathrm{HC} 4$ & ACID1/worst \\
\hline Ex2_1_7 & 20 & 10 & 8.75 & 465 & 3 & $\mathrm{HC} 4$ & 3BCID-fp & 1.03 & 1.03 & 0.7 \\
\hline Ex2_1_8 & 24 & 10 & 6.18 & 200 & 0 & $\mathrm{HC} 4$ & $3 B C I D-f p$ & 1.06 & 1.06 & 0.91 \\
\hline Ex2_1_9 & 10 & 1 & 10.1 & 1922 & 0.75 & $\mathrm{HC} 4$ & 3BCID-fp & 1.04 & 1.04 & 0.9 \\
\hline Ex5_4_4 & 27 & 19 & 915 & 23213 & 0.8 & ACID1 & $3 B C I D-n$ & 1 & 0.96 & 0.91 \\
\hline Ex6_1_1 & 8 & 6 & 60.8 & 13071 & 8.9 & $\mathrm{HC} 4$ & $3 B C I D-f p$ & 1.21 & 1.21 & 0.73 \\
\hline Ex6_1_3 & 12 & 9 & 297 & 29154 & 11.7 & $\mathrm{HC} 4$ & $3 B C I D-f p$ & 1.19 & 1.19 & 0.63 \\
\hline Ex6_1_4 & 6 & 4 & 1.99 & 505 & 6 & ACID1 & $3 B C I D-f p$ & 1 & 0.97 & 0.8 \\
\hline Ex6_2_6 & 3 & 1 & 107 & 46687 & 0 & $\mathrm{HC} 4$ & $3 B C I D-f p$ & 1.02 & 1.02 & 0.74 \\
\hline Ex6_2_8 & 3 & 1 & 48.2 & 21793 & 0.1 & $\mathrm{HC} 4$ & $3 B C I D-f p$ & 1.01 & 1.01 & 0.72 \\
\hline Ex6_2_9 & 4 & 2 & 51.9 & 19517 & 0.1 & $\mathrm{HC} 4$ & $3 B C I D-f p$ & 1.02 & 1.02 & 0.72 \\
\hline Ex6_2_10 & 6 & 3 & 2248 & 569816 & 0 & ACID1 & $3 B C I D-f p$ & 1 & 0.99 & 0.64 \\
\hline Ex6_2_11 & 3 & 1 & 29.3 & 13853 & 0.3 & $\mathrm{HC} 4$ & 3BCID-fp & 1.05 & 1.05 & 0.73 \\
\hline Ex6_2_12 & 4 & 2 & 21.6 & 7855 & 0.1 & $\mathrm{HC} 4$ & $3 B C I D-f p$ & 1.02 & 1.02 & 0.8 \\
\hline Ex7_2_3 & 8 & 6 & 19.4 & 4596 & 4.4 & $3 B C I D-n$ & $\mathrm{HC} 4$ & 1.07 & 0.17 & 0.17 \\
\hline Ex7_2_4 & 8 & 4 & 36.8 & 5606 & 4.2 & $3 B C I D-f p$ & $\mathrm{HC} 4$ & 1.04 & 0.66 & 0.66 \\
\hline Ex7_2_8 & 8 & 4 & 38.0 & 6792 & 4.1 & $3 B C I D-n$ & $\mathrm{HC} 4$ & 1.09 & 0.71 & 0.71 \\
\hline Ex7_2_9 & 10 & 7 & 78.0 & 14280 & 9.3 & $3 B C I D-n$ & $\mathrm{HC} 4$ & 1.07 & 0.48 & 0.48 \\
\hline Ex7_3_4 & 12 & 17 & 2.95 & 366 & 3 & $3 B C I D-n$ & 3BCID-fp & 1.23 & 0.99 & 0.89 \\
\hline Ex7_3_5 & 13 & 15 & 4.59 & 894 & 6 & $3 B C I D-n$ & $\mathrm{HC} 4$ & 1.05 & 0.38 & 0.38 \\
\hline Ex8_4_4 & 17 & 12 & 1738 & 46082 & 0.9 & ACID1 & 3BCID-fp & 1 & 0.99 & 0.87 \\
\hline Ex8_4_5 & 15 & 11 & 772 & 25454 & 4.8 & $\mathrm{HC} 4$ & $3 B C I D-f p$ & 1.03 & 1.03 & 0.75 \\
\hline Ex8_5_1 & 6 & 5 & 9.67 & 2138 & 2.75 & ACID1 & $3 B C I D-f p$ & 1 & 0.84 & 0.82 \\
\hline Ex8_5_2 & 6 & 4 & 32.5 & 5693 & 0.8 & ACID 1 & $3 B C I D-f p$ & 1 & 0.9 & 0.87 \\
\hline Ex8_5_6 & 6 & 4 & 32.4 & 10790 & 1.8 & $\mathrm{HC} 4$ & $3 B C I D-f p$ & 1.02 & 1.02 & 0.76 \\
\hline Ex14_1_7 & 10 & 17 & 665 & 95891 & 3.3 & $3 B C I D-n$ & $\mathrm{HC} 4$ & 1.03 & 0.61 & 0.61 \\
\hline Ex14_2_3 & 6 & 9 & 2.01 & 360 & 2 & $\mathrm{HC} 4$ & $3 B C I D-f p$ & 1.17 & 1.17 & 0.69 \\
\hline Ex14_2_7 & 6 & 9 & 49.9 & 5527 & 0 & $\mathrm{HC} 4$ & $3 B C I D-n$ & 1.47 & 1.47 & 0.48 \\
\hline alkyl & 14 & 7 & 3.95 & 714 & 4 & $\mathrm{HC} 4$ & $3 B C I D-f p$ & 1.2 & 1.2 & 0.91 \\
\hline bearing & 13 & 12 & 11.6 & 1098 & 13 & $3 B C I D-n$ & $\mathrm{HC} 4$ & 1.01 & 0.53 & 0.53 \\
\hline hhfair & 28 & 25 & 26.6 & 3151 & 10 & $3 B C I D-n$ & $\mathrm{HC} 4$ & 1.12 & 0.58 & 0.58 \\
\hline himmel16 & 18 & 21 & 188 & 21227 & 15.5 & $3 B C I D-n$ & 3BCID-fp & 1.1 & 0.94 & 0.88 \\
\hline house & 8 & 8 & 62.8 & 27195 & 3.25 & $\mathrm{HC} 4$ & $3 B C I D-f p$ & 1.09 & 1.09 & 0.79 \\
\hline hydro & 30 & 24 & 609 & 32933 & 0 & ACID1 & $3 B C I D-f p$ & 1 & 0.88 & 0.78 \\
\hline immun & 21 & 7 & 4.17 & 1317 & 2.5 & ACID1 & $3 B C I D-f p$ & 1 & 0.55 & 0.28 \\
\hline launch & 38 & 28 & 107 & 2516 & 21 & ACID1 & $3 B C I D-n$ & 1 & 0.79 & 0.43 \\
\hline linear & 24 & 20 & 751 & 27665 & 0.25 & ACID1 & $3 B C I D-n$ & 1 & 0.98 & 0.65 \\
\hline meanvar & 7 & 2 & 2.43 & 370 & 2 & $\mathrm{HC} 4$ & $3 B C I D-f p$ & 1.04 & 1.04 & 0.84 \\
\hline process & 10 & 7 & 2.61 & 611 & 8 & $\mathrm{HC} 4$ & $3 B C I D-f p$ & 1.08 & 1.08 & 0.77 \\
\hline ramsey & 31 & 22 & 164 & 4658 & 4.3 & ACID1 & $3 B C I D-f p$ & 1 & 0.85 & 0.68 \\
\hline srcpm & 38 & 27 & 160 & 6908 & 0.5 & ACID1 & $3 B C I D-f p$ & 1 & 0.62 & 0.33 \\
\hline
\end{tabular}

Table IV

OPTIMIZATION PROBLEMS: GAIN RATIO IN SOLVING TIME: TIME ACID1/TIME XXX

\begin{tabular}{|l|r|r|r|r|r|r|}
\hline & ACID1 & \multicolumn{1}{|c|}{ HC4 } & 3BCID-fp & 3BCID-n & ACID 0 & ACID2 \\
\hline \hline \#solved instances & 40 & 40 & 40 & 40 & 40 & 40 \\
\hline Average gain & 1 & 0.9 & 0.77 & 0.88 & 0.91 & 0.97 \\
\hline Maximum gain & 1 & 0.17 & 0.28 & 0.35 & 0.62 & 0.28 \\
\hline Maximum loss & 1 & 1.47 & 1.04 & 1.23 & 1.18 & 1.19 \\
\hline Standard deviation gain & 0 & 0.25 & 0.16 & 0.18 & 0.12 & 0.14 \\
\hline Total time & 9380 & 10289 & 12950 & 11884 & 11201 & 9646 \\
\hline Total gain & 1 & 0.91 & 0.72 & 0.79 & 0.84 & 0.97 \\
\hline
\end{tabular}

